\title{
Analisi critica su le profondità ipocentrali.
}

(Critical analysis about the hypocentral depths)

\author{
L. Marcelli* ${ }^{*}$ M. Peronaci**
}

Ricevuto il 21 Dicembre 1979

\begin{abstract}
The aim of this study is to point out the difficulties in the analytical determination of hypocentral depth $h$ and the consequent uncertainty in its reliability.

The former attempt was realized at world-wide level (december 1964), the latter in the Mediterranean Area (19 reigons, accordingly to the division proposed by Flin, Engdal, Hill, 1974).

We considered hypocentral data of the earthquakes occurred in that area during the 1964-1974 decade, with a great care to the $h$ values and their standard deviations.

First we pointed out the depth features of every region: afterwards we looked into the standard deviations $(\varepsilon)$ and their respective $h$ values

Data analysis suggested us to calculate a series of exponential functions $\varepsilon=\beta h^{-\alpha}$ showing standard deviations as a function of focal depth; every region is characterized by $\alpha$ and $\beta$ coefficients calculated by a least squares fit.
\end{abstract}

(*) Istituto Nazionale di Geofisica.

(**) Borsista del CNEN. 
We analysed results and also made an attempt to explain the standard deviations scatter. We also made an attempt by only Italian earthquakes data (ioining the four sections Northern, Central, Southern Italy, and Sicily), and we calculated the $\varepsilon=f(h)$ function.

Both the world-wide analysis and the Mediterranean one pointed out some interesting elements.

- Focal depth data, even if joined with their standard deviations, are very unrealiable particularly for crustal earthquakes; in that case standard deviations often are too large and focal depth lose their physical meaning.

- In the upper mantle the data are more reliable.

Obviously the choise of the travel-times used to calculate focal depth influences data reliability, particularly in the most heterogeneous layers in the crust.

\section{RIASSUNTO}

Il presente studio si propone di mettere in evidenza le difficoltà che si incontrano nella determinazione analitica del parametro ipocentrale $h$ e la conseguente incertezza che ne deriva per la bontà del dato stesso.

Dopo un primo sondaggio condotto a livello mondiale (per un arco di tempo molto limitato) l'indagine è stata ristretta all'area Mediterranea (diciannove regioni - secondo la suddivisione di Flinn, Engdahl, Hill le più sismicamente attive) allargando i termini di tempo. Sono stati esaminati $\mathrm{i}$ dati ipocentrali di tutti i terremoti avvenuti in detta area nel decennio 1964-74, prendendo però in esame solo quelli di cui era stata calcolata la profondità ipocentrale, con particolare attenzione per le $h$ accompagnate dall'errore standard.

Sono state messe dapprima in rilievo le caratteristiche di profondità delle singole regioni inquadrate nell'intera area Mediterranea; si è passati poi all'esame dettagliato degli errori $(\bar{F})$ associati alle $h$.

L'analisi ha condotto al calcolo di una serie di curve esponenziali del tipo $\vec{\varepsilon}=\beta h^{-\alpha}$ rappresentative dell'andamento medio degli errori in funzione del valore delle profondità ipocentrali; ogni regione risulta caratterizzata da valori di $\alpha$ e $\beta$ desunti dall'analisi matematica condotta.

I risultati sono stati analizzati in dettaglio ed è stato fatto anche un tentativo di interpretazione per quanto riguarda la dispersione dei singoli errori standard intorno ai valori mediati rappresentati dalle curve.

Un ulteriore studio è stato fatto restringendo il problema alla sola Italia (unificandone le sezioni principali Nord-Centro-Sud-Sicilia) in un quadro unico. Anche per l'Italia (così considerata) è stata calcolata la curva $\vec{\varepsilon}=f(h)$ e se ne sono discussi i risultati. 
Sia l'analisi mondiale, sia le analisi dettagliate riguardanti le singole regioni e successivamente la sola Italia, hanno messo in luce degli elcmenti interessanti che, pur diversificandosi nei particolari da regione a regione, hanno delle caratteristiche comuni.

(e scelta delle regioni sismiche da esaminare) standard, è da considerarsi molto incerto, specie per quanto riguarda i terremoti crostali: da questi ipocentri ci si possono aspettare errori talmente elevati da far perdere al dato calcolato qualunque significato fisico.

- Le cose vanno meglio nel mantello superiore e in generale per tutti i terremoti con ipocentro più profondo del normale.

E' evidente l'influenza che gioca nel calcolo delle profondità la scelta dei modelli di dromocrone usate, tanto più diversificati l'uno dall'altro quanto più eterogenea è la struttura degli strati interni attraversati dalle onde sismiche.

Tabelle e grafici illustrano i vari punti del problema.

CONSIDERAZIONI GENERALI SU LE PROFONDITÀ IPOCENTRALI DEI TERREMOTI E GLI ERRORI DI CALCOLO ASSOCIATI ALLA LORO DETERMINAZIONE

\section{Richiami}

In un precedente lavoro (Marcelli, Pannocchia, 1974), sono state messe in evidenza le gravi difficoltà che si incontrano nella determinazione dei parametri ipocentrali $(\alpha, \lambda, H, h)$ di un qualsiasi evento sismico, dedotti come d'uso, dai tempi d'arrivo delle onde longitudinali nei vari Osservatori del mondo. Si è visto come i calcoli, eseguiti mediante elaboratori elettronici, conducano a determinare con rigore matematico un punto all'interno della terra $(\varphi, \alpha, h)$ ed un tempo origine $H$; a ciascuno dei quattro parametri è associato un errore standard desunto analiticamente.

Si è visto come e quanto importanti siano tali errori e quanto essi possano influenzare la stima delle distanze epicentrali, specialmente per quanto riguarda gli errori $\delta \varphi, \delta \lambda, \quad \delta H$ associati rispettivamente all'epicentro $(\varphi, \lambda)$ e al tempo origine $H$.

Nel presente lavoro si vuol porre l'attenzione sul quarto 
parametro ipocentrale, la profondità, la cui determinazione è, come ognuno sa, estremamente complessa. Questo parametro, come anche gli altri, viene calcolato mediante $\mathrm{i}$ tempi di arrivo delle onde longitudinali, ossia tramite la velocità di propagazione delle onde sismiche, che obbediscono ad un modello matematico di propagazione di onde elastiche.

Dalla teoria dell'elasticità si sa che la velocità di propagazione delle onde in un miezzo elastico dipende essenzialmente dalle due costanti di Lamé e dalla densità del mezzo elastico. Però la densità del mezzo-Terra a sua volta varia, insieme con diversi altri parametri (pressione, temperatura, composizione chimica e fase) anch'essi variabili all'interno della Terra in maniera estremamente complessa, al variare della profondità misurata lungo il raggio terrestre; e tale legge di variazione, stabilita per alcuni modelli riferentisi alle sette stratificazioni principali A, B, C, D, E, F, G inizialmente proposte da Bullen (Bullen, 1975) omogenee e concentriche all'interno della Terra, fa agevolmente intuire quanto influisca il valore di $h$ sulla velocità di propagazione. Se poi si pensa che il modello " $A$ » che rappresenta la crosta terrestre è indubbiamente il più eterogeneo ed il più irregolare, dai terremoti con ipocentro entro la crosta ci si possono aspettare le maggiori anomalie.

CAP. 1. - SCOPO DELla Ricerca

Un primo sondaggio orientativo (a livello mondiale)

Nel presente lavoro ci proponiamo di quantizzare questi concetti, sia per porre in evidenza l'importanza di conoscere gli errori standard associati ai valori delle profondità ipocentrali calcolate, sia per tentare una interpretazione degli errori stessi, qualora questo fosse possibile.

A questo scopo, per una prima indagine orientativa, abbiamo esaminato la documentazione delle profondità ipocentrali quali risultano dal Catalogo Regionale pubblicato dall'I.S.C. (1964-1974). 
Ci riferiremo quasi sempre ad esso per avere la certebba di un punto di riferimento costante sia per la metodologia dei calcoli sia per l'omogeneità dei dati di Osservatorio che ad esso affluiscono. Solo eccezionalmente verranno usati dati forniti da altre organizzazioni presi con il seguente ordine di preferenza: BCIS, USCGS, MOSCA, ATENE.

Questa prima indagine campione esplorativa è stata condotta sui dati di tutto il mondo, considerati entro un breve arco di tempo (dicembre 1974). E' ovvio che tale scelta non è idonea a puntualizzare il problema, tuttavia essa è servita a mettere in evidenza i seguenti punti che ci sono sembrati piuttosto importanti e che ritroveremo, più precisati, in una indagine più accurata:

- la maggior parte dei terremoti posti a profondità $h=0$ viene presentata senza errore;

- gli errori associati alle piccole profondità sono per lo più talmente elevati da far perdere ogni significato al dato di $h$ (esempi: $h=0 \pm 12 ; h=2 \pm 20 ; h=5 \pm 31 ; h=11 \pm 26 \ldots$ );

- in generale si è potuto rilevare che anche quando vengono dati valori numerici di $h$ molto piccoli $(\leq 10 \mathrm{~km}), l^{\prime} 85 \%$ di essi è privo di errore.

(Quanto riguarda le piccole profondità, si può attribuire in parte alla scarsità di dati di osservazioni sperimentali quando si tratta di terremoti di piccola intensità; però molto spesso l'errore è elevatissimo anche con numerosi dati di osservazione.

Va tenuto presente a questo punto che ogni metodo analitico seguito per la determinazione della profondità assimila il fuoco sismico ad un punto geometrico; l'inesattezza di questa assunzione porta ad un errore sulla $h$ che può ritenersi dipendente dalle dimensioni geometriche del volume interessato dal fuoco sismico e conseguentemente l'errore percentuale $(\varepsilon=\mathrm{d} h / h \%)$ sarà tanto più elevato quanto più piccolo è il valore della profondità ipocentrale; però è da ritenere che non sempre questo possa giustificare errori così notevoli quali quelli evidenziati). 
- una lunga serie di dati di profondità privi di errore si trova anche a $33 \mathrm{~km}$ che è la profondità media convenzionale della crosta terrestre.

- sotto la Crosta, nel Mantello Superiore, gli errori associati alle profondità sono notevolmente minori, pur mantenendosi ancora piuttosto elevati, almeno fino a $130 \mathrm{~km}$ di profondità circa.

- per terremoti con ipocentro nel Mantello Inferiore le cose vanno decisamente meglio: gli errori relativi in percentuale raggiungono valori molto bassi.

Questa semplice e sommaria indagine, pur non avendo consistenza per la scarsità degli elementi presi in considerazione, può già dare tuttavia una prima indicazione sommaria così riassumibile:

1) sono evidenti le difficoltà di ottenere dai calcoli generalizzati, valori di profondità sufficientemente precisi, specialmente nella Crosta dove le eterogeneità strutturali sono marcate;

2) là dove il tragitto delle onde sismiche si svolge prevalentemente in mezzi pressoché omogenei gli errori di calcolo si riducono notevolmente;

3) l'analisi delle profondità nella Crosta e nel Mantello superiore presenta una ulteriore caratteristica: se si vuol delineare un qualche collegamento tra l'andamento degli errori relativi medi e le profondità, si nota una dispersione talmente grande nei valori dei singoli scarti relativi ad una stessa profondità, che la media stessa perde significato. Ad esempio per vari terremoti a profondità $h=49 \mathrm{~km}$ sono stati dati errori diversi, e questo sarebbe ragionevole provenendo essi da zone diverse, ma il « range » degli errori è troppo esteso per poterlo ritenere accettabile (esso va da 4,7 a $\pm 40 \mathrm{~km}$ ).

Gli spunti di osservazione sono molti ed interessanti, ma non conviene dilungarsi troppo sul problema a scala mondiale che potrebbe risultare dispersivo in quanto non si può ipotizzare su un modello a scala generale. 
CAP. 2 - Il problema Ristretto all'area MisdtTrRRanea

Analisi delle h dei terremoti con ipocentro in detta area.

(Tempo di indagine: il decennio 1964-1974).

Da quanto precede risulta evidente l'opportunità di affrontare il problema considerando un intervallo di tempo ragionevolmente esteso onde acquisire dati sufficienti per una analisi statistica, ma è altrettanto opportuno restringere l'area di ricerca in modo da ridurre le eterogeneità strutturali della crosta.

Per rendere il problema più adatto ad un interesse che investa più da vicino il territorio nazionale, abbiamo ristretto la ricerca alla sola area Mediterranea, proponendoci una ulteriore successiva analisi per l'Italia in particolare. Poiché tuttavia l'Italia fa parte di un sistema strutturale che si inserisce nell'area Mediterranea abbiamo scelto quest'ultima per poter porre a confronto i dati particolari italiani con quelli più generali del bacino del Mediterraneo.

A tale scopo ci siamo serviti della suddivisione mondiale in cinquanta regioni sismiche proposta inizialmente da Gutenberg e Richter, successivamente frazionata da Flinn, Engdahl e Hill (Flinn, Engdahl, 1974). Tale suddivisione è quella adottata dall'I.S.C. per il suo Catalogo Regionale.

Abbiamo inizialmente considerato le seguenti regioni geografiche che abbracciano tutta l'area Mediterranea:

Portogallo, Stretto di Gibilterra, Spagna, Pirenei, Francia Meridionale, Francia, Corsica, Italia Settentrionale, Italia Centrale, Italia Meridionale, Sicilia, Sardegna, Mar Tirreno, Mare Adriatico, Mare Jonio, Austria, Jugoslavia, Albania, Grecia-Albania, Grecia, Grecia Meridionale, Dodecanneso, Creta, Mar Egeo, Turchia, Turchia-URSS, Iraq, Cipro, Giordania-Siria, Egitto, Mar Morto, Libia (coste), Algeria, Tunisia, Marocco, Mediterraneo, Mediterraneo Occidentale, Mediterraneo Orientale.

Per quanto riguarda il periodo di tempo cui è stata estesa l'indagine, si è ritenuto opportuno prendere l'avvio dal mo- 
mento in cui l'I.S.C. ha iniziato i suoi calcoli con l'elaborazione elettronica dei dati di osservazione trasmessigli dai vari Osservatori, e precisamente a partire dal $1^{\circ}$ gennaio 1964 . Pertanto il periodo investigato copre il decennio 1964-1974.

Dato lo scopo della presente ricerca sono stati presi in esame solo i terremoti per i quali l'I.S.C. ha fornito i valori di $h$; ne segue che l'andamento della sismicità che abbiamo cercato di evidenziare (in rapporto alle profondità ipocentrali) non ne è la rappresentazione fedele in quanto vengono trascurati i terremoti di scarsa intensità che sono generalmente quelli per $i$ quali non è stato fornito il parametro di profondità; riteniamo però che questa incompletezza non debba nuocere ad una analisi dei dati raccolti lungo un arco di tempo sufficientemente esteso quale quello esaminato.

Considerazioni generali sulla sismicità dell'area Mediterranea

(e scelta delle regioni sismiche da esaminare)

Dobbiamo premettere che una analisi generale della sismicità condotta su tutte le sezioni geografiche elencate al punto precedente conferma quanto già noto; esse non sono tutte attive alla stessa maniera anche se va tenuto presente che alcuni paesi non dispongono ancora di reti sismiche nazionali in grado di fornire osservazioni sufficienti a determinare i parametri ipocentrali: conseguentemente $i$ terremoti di scarsa intensità, se ci sono, sfuggono alle raccolte mondiali.

La nostra indagine si è pertanto ulteriormente ristretta alle seguenti 19 sezioni che sono risultate le più documentate nel decennio considerato:

sez. sez.

Italia Settentrionale

Grecia-Albania

Italia Centrale

Grecia

Italia Meridionale

Grecia Meridionale 
Sicilia

Dodecanneso

Mar Adriatico

Mar Egeo

Mar Jonio

Creta

Mar Tirreno

Mediterraneo Orientale

Turchia

Jugoslavia

Algeria

Albania

Stretto di Gibilterra

\section{Caratteristiche di profondità}

Un primo esame è stato condotto nel modo seguente: di ogni sezione sono stati raggruppati tutti i terremoti in classi di profondità intervallate di 5 in $5 \mathrm{~km}$ e se ne sono tracciati gli istogrammi di Figg. 1/1 ..1/19 (in ascisse sono riportate le varie classi di profondità e in ordinate il numero degli eventi corrispondenti). Solo per la Turchia, a titolo di esemplificazione utile per una analisi più dettagliata, le profondità sono state riportate di $\mathrm{km}$ in $\mathrm{km}$. Per le altre regioni i valori $h=2.5,7.5$, $12.5 \mathrm{~km}, \ldots$ rappresentano la media degli $n$ valori di $h$ compresi negli intervalli $h_{i} \pm 2.5 \mathrm{~km}\left({ }^{*}\right)$. Abbiamo scelto la la scala delle profondità con passo di $5 \mathrm{~km}$ per evitare di avere troppi sottoinsiemi di $h$, consapevoli che tale "lisciamento " non avrebbe compromesso troppo il significato fisico dei risultati; una risoluzione maggiore, quale quella usata per la Turchia, perderebbe significato nella definizione degli errori percentuali medi e sarebbe di scarso interesse in uno studio a carattere conoscitivo come il presente.

La tabella 1 riassume gli elementi principali di questa ricerca.

(*) $h_{i}$ sono multipli interi di $h=5 \mathrm{~km}$.

Per il Mar Tirreno, sede di eventi profondi, è stata fatta una suddivisione diversa (vedi grafico).

Per la Turchia riportiamo, per semplicità, solo il grafico. 


$$
\begin{aligned}
& f=-1= \\
& =-1=-1=
\end{aligned}
$$

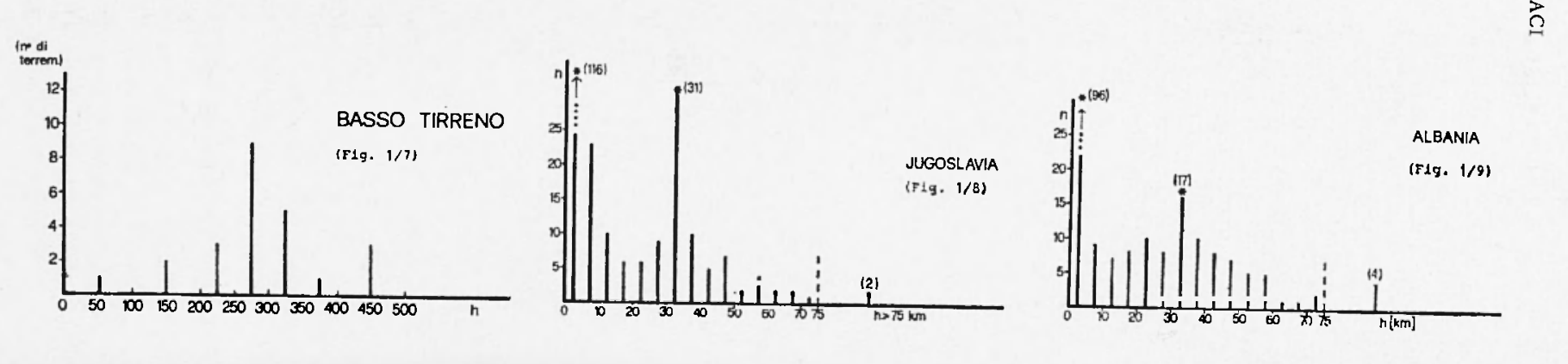



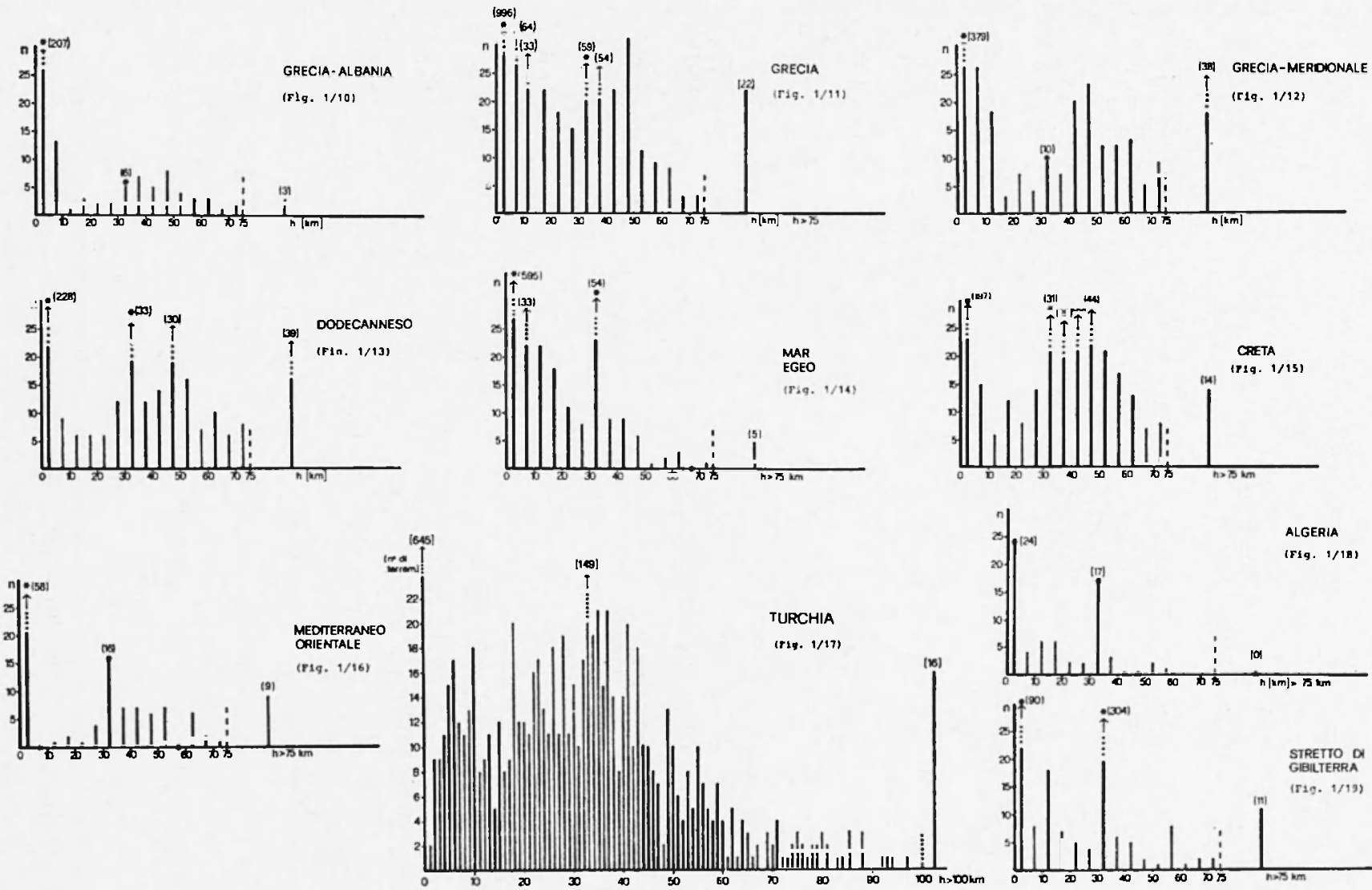

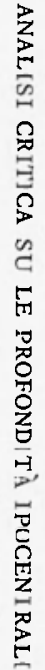

Figg. $1 / 1 \ldots 1 / 19$ - Serie degli istogrammi per tutte le re gioni considerate: distribuzione della frequenza in classi di profondità. Distribuzione delle profondità ipocentrali nelle regioni dell'area Mediterranea. 
Per ogni regione geografica esaminata sono riportati i dati numerici relativi alle seguenti indicazioni:

numero totale $(N)$ dei terremoti avvenuti nei 10 anni esaminati

numero dei terremoti provenienti da uguali profondità ipocentrali (raggruppate ad intervalli di $5 \mathrm{~km}$ come sopra detto)

per ciascun intervallo di profondità, insieme con la percentuale dei terremoti appartenenti ad esso, è indicato anche il numero dei terremoti dati "senza" errore e il numero di quelli dati «con»l'errore $(h+\delta h)$

$i$ terremoti $\operatorname{con} h>75 \mathrm{~km}$ sono stati considerati tutti insieme ad esclusione di quelli del basso Tirreno che sono i soli, nella intera area considerata, ad avere profondità notevolmente elevate $(100<h<500 \mathrm{~km})$

per it Mar Tirreno è stata fatta una tabella a parte (tabella $1 b)$.

L'esame della tabella 1 insieme con l'esame degli istogrammi corrispondenti offre una panoramica molto significativa per il problema posto in questa ricerca.

La maggior parte dei terremoti sembra provenire dalla profondità di $h \leq 5 \mathrm{~km} \mathrm{e}$, in numero molto minore, da una profondità compresa tra 30 e $35 \mathrm{~km}$.

E' una realtà fisica, o un risultato fittizio dovuto alla difficoltà di calcolare le profondità ipocentrali? Lo Stretto di Gibilterra ad esempio e, tra tutte le regioni, quella che ha il maggior numero di terremoti a profondità $33 \mathrm{~km}$ (in numero di 309) però essi sono tutti senza errore!

Nel paragrafo seguente discuteremo sul problema degli errori associati al calcolo delle profondità.

Per il momento possiamo dire che il quadro delle tabelle la e lb e l'insieme dei grafici 1 , conduce alle due seguenti osservazioni principali :

1) quanto alla sismicità: delle regioni esaminate le più sismicamente attive sono risultate nel decennio considerato la Turchia (con 1.534 terremoti) e la Grecia (con 1.370 terremoti): la più scarsamente attiva il Mar Adriatico (con soli 35 terre- 
TABELLA 1a

\begin{tabular}{|c|c|c|c|c|c|c|c|c|c|c|c|c|c|c|c|c|c|c|c|c|c|c|c|c|c|c|c|c|c|c|c|c|c|c|c|c|c|c|c|c|c|c|c|c|c|c|c|c|c|c|}
\hline \multirow{2}{*}{ Regione } & \multirow{2}{*}{$\begin{array}{c}\text { Sez. } \\
\text { n. }\end{array}$} & \multirow{2}{*}{ 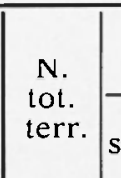 } & \multicolumn{3}{|c|}{$0<h<5$} & \multicolumn{3}{|c|}{$5<h<10$} & \multicolumn{3}{|c|}{$10<h<15$} & \multicolumn{3}{|c|}{$15<h<20$} & \multicolumn{3}{|c|}{$20<h<25$} & \multicolumn{3}{|c|}{$25<h<30$} & \multicolumn{2}{|c|}{$30<h<35$} & \multicolumn{3}{|c|}{$35<h<40$} & \multicolumn{3}{|c|}{$40<h<45$} & \multicolumn{3}{|c|}{$45<h<50$} & \multicolumn{3}{|c|}{$50<h<55$} & & $<h<60$ & & & $<h<1$ & & & $<h<7$ & & & $<n<$ & & & $h>75$ & & \\
\hline & & & senza & con $\mid$ & $\%$ & senza| & con & $\%_{0}$ & senzal & con & $\%$ & sen. & con $\mid$ & $\%$ & sen. & con & $\%$ & sen. & con $\mid$ & \begin{tabular}{l|l}
$\%$ & $\mathrm{ss}$
\end{tabular} & $\begin{array}{l}\text { sen. } \\
\text {. c }\end{array}$ & con ${ }^{\circ}$ & $\begin{array}{lll}\% & \text { sen. }\end{array}$ & $\begin{array}{l}\text { n. } \\
\mid \text { con } \mid\end{array}$ & \begin{tabular}{|c|} 
\\
\end{tabular} & sen. & con & $\%$ & sen. & con & $\%$ & sen.. & con $\mid$ & $\begin{array}{lll}\% & \mathrm{~s} \\
\%\end{array}$ & $\begin{array}{l}\text { sen. } \\
\text { | c }\end{array}$ & con $\mid$ & $\%$ & sen. & con & $\%$ & sen. & con & $\%=$ & sen. & con & $\%$ & sen. & con & $\%$ & Regione \\
\hline ia Settentrionale & 545 & 203 & 130 & 363 & 5.52 & - & 9 & 4.43 & 1 & 2 & 1.49 & 1 & 3 & 1.97 & 3 & $\begin{array}{ll}3 & 2 \\
3\end{array}$ & 2.96 & - & \begin{tabular}{l|l}
4 & 1 \\
\end{tabular} & 1.97 & 18 & \begin{tabular}{l|l}
3 & 10. \\
\end{tabular} & \begin{tabular}{l|l}
0.30 & - \\
-
\end{tabular} & 3 & $\mid 1.49$ & 2 & 3 & 2.5 & - & \begin{tabular}{l|l}
6 & 2 \\
\end{tabular} & 2.95 & - & \begin{tabular}{l|l}
1 & 0 \\
\end{tabular} & 0.49 & 1 & $\begin{array}{ll}3 & 1 \\
3\end{array}$ & & - & 1 & 0.49 & - & 1 & 0.49 & - & - & - & - & & 0.98 & Italia Set \\
\hline ia Centrale & 381 & 154 & 77 & & 5.19 & - & 6 & 3.90 & - & 7 & 4.54 & 1 & $4: 3$ & 3.25 & 2 & \begin{tabular}{l|l}
4 & 3 \\
\end{tabular} & 3.90 & 1 & \begin{tabular}{l|l}
2 & 1
\end{tabular} & 1.95 & 21 & & \begin{tabular}{l|ll}
4.28 & 1 &
\end{tabular} & 2 & 1.95 & 1 & 6 & 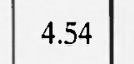 & - & & 1.30 & - & - & - & - & \begin{tabular}{l|l}
2 & 1 \\
$Y$
\end{tabular} & 1.30 & - & - & - & - & & 1.30 & - & 2 & 1.30 & - & & 1.30 & Italia Centra \\
\hline ia Meridionale & 390 & 74 & 39 & \begin{tabular}{l|l|l|l|} 
&
\end{tabular} & 4.05 & 4 & -1 & 5.40 & 1 & 1 & 2.70 & -1 & 2 & 2.70 & 1 & \begin{tabular}{l|l}
2 & 4
\end{tabular} & 4.05 & - & -1. & - & 7 & & $0.81-$ & 5 & \begin{tabular}{|l|} 
\\
\end{tabular} .77 \mid & - & 2 & 2.70 & - & \begin{tabular}{l|l}
1 & 1
\end{tabular} & 1.35 & - & \begin{tabular}{l|l}
3 & 4 \\
\end{tabular} & 4.05 & - & -1 & - & - & -1 & - & - & 1 & 1.35 & - & - & - & - & & 4.05 & Italia Meridionale \\
\hline & 398 & 51 & 15 & \begin{tabular}{l|l|l|}
3 & $3:$
\end{tabular} & 35.29 & - & -1 & -1 & 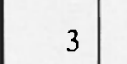 & 1 & 7.84 & - & 1 & 1.96 & - & 59 & 9.80 & - & \begin{tabular}{l|l}
2 & 3 \\
\end{tabular} & 3.92 & 4 & \begin{tabular}{l|l}
2 & $11: 2$
\end{tabular} & \begin{tabular}{l|l}
1.76 & - \\
-
\end{tabular} & 6 & 11.77 & 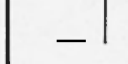 & 2 & \begin{tabular}{|l|}
3.92 \\
\end{tabular} & - & $3: 5$ & 5.88 & 2 & \begin{tabular}{l|l}
-3 \\
-1
\end{tabular} & 3.92 & - & \begin{tabular}{l|l}
2 & 3
\end{tabular} & 3.92 & - & - & - & - & - & - & - & - & - & - & - & - & \begin{tabular}{|l|} 
Sicilia \\
\end{tabular} \\
\hline tico & 382 & 35 & 19 & $\left.2\right|_{6}$ & 60.00 & - & - & - & - & 1 & 2.86 & - & - & - & 1 & \begin{tabular}{l|l}
2 & 8
\end{tabular} & 8.57 & - & -1. & 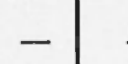 & - & -1 & -1 & 2 & 5.71 & -1 & 2 & 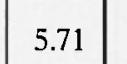 & 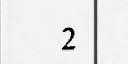 & -5 & 5.71 & - & \begin{tabular}{l|l}
1 & 2
\end{tabular} & 2.86 & - & \begin{tabular}{l|l}
1 & 2 \\
\end{tabular} & 2.86 & - & 1 & 2.86 & - & - & - & - & 1 & 2.86 & - & - & - & Mar Adriatico \\
\hline Ic & 399 & 267 & 178 & \begin{tabular}{l|l}
13 & $7_{11}$
\end{tabular} & & 1 & 12 & 4.87 & - & 8 & 3.00 & -1 & 2 & 0.75 & 3 & $\begin{array}{lll}4 & 2\end{array}$ & 2.62 & - & \begin{tabular}{l|l}
3 & 1
\end{tabular} & 1.12 & $=$ & \begin{tabular}{l|l}
3 & 3.4
\end{tabular} & 3.00 & 10 & 4.12 & - & 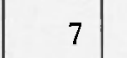 & $2.62 \mid$ & 1 & 4 & 1.87 & - & \begin{tabular}{l|l}
3 & 1
\end{tabular} & 1.12 & - & \begin{tabular}{l|l}
4 & 1
\end{tabular} & 1.50 & - & 1 & 0.37 & -1 & & \begin{tabular}{|l|l|} 
& \\
\end{tabular} & - & - & | & -1 & 2 & $\mid$\begin{tabular}{|c|c|}
$\mid$ & 0.75
\end{tabular} & \begin{tabular}{|l} 
Mare Ionio \\
\end{tabular} \\
\hline & \begin{tabular}{|l|}
396 \\
\end{tabular} & 67 & 22 & $\begin{array}{ll}2 & 35\end{array}$ & & 1 & 3 & 5.97 & 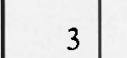 & 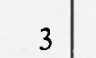 & 8.95 & 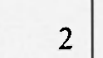 & 4 & 8.95 & - & $\begin{array}{ll}2 & 2\end{array}$ & 2.98 & - & $\begin{array}{ll}2 & 2\end{array}$ & 2.98 & 14 & $\begin{array}{ll}3 & 25 .\end{array}$ & $5.37-$ & 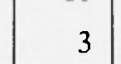 & 4.48 & - & - & 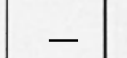 & - & -1 & - & - & $\begin{array}{ll}2 & 2\end{array}$ & 2.98 & - & \begin{tabular}{l|l}
1 & 1
\end{tabular} & 1.49 & - & -1 & -1 & -1 & - & - & -1 & -1 & - & -1 & - & -1 & \begin{tabular}{|l|l|} 
Algeria \\
\end{tabular} \\
\hline & \begin{tabular}{|l|}
364 \\
\end{tabular} & 1370 & 941 & \begin{tabular}{l|l}
55 & 72
\end{tabular} & 2.70 & 2 & 62 & 4.67 & 4 & ${ }_{29}$ & 2.41 & 2 & 20 & 1.61 & 1 & $\begin{array}{lll}17 & 1 \\
17\end{array}$ & 1.31 & - & \begin{tabular}{l|l}
15 & 1.
\end{tabular} & 1.09 & 26 & \begin{tabular}{l|l}
33 & 4. \\
\end{tabular} & 4.31 & 53 & \begin{tabular}{|l|}
3.94 \\
\end{tabular} & - & 22 & 1.61 & 7 & \begin{tabular}{l|l}
24 & 2 \\
2
\end{tabular} & 2.26 & - & \begin{tabular}{l|l}
111 & 0
\end{tabular} & 0.80 & - & \begin{tabular}{l|l}
9 & 0
\end{tabular} & 0.66 & 1 & 7 & 0.58 & -1 & 3 & 0.22 & -1 & 3 & 0.22 & 3 & 19 & $\mid 1.61$ & Grecia \\
\hline anneso & \begin{tabular}{|l|}
369 \\
\end{tabular} & 442 & 221 & $\begin{array}{lll}7 & 5\end{array}$ & & - & $\begin{array}{r}0 . \\
9\end{array}$ & 2.04 & - & 6 & 1.36 & - & 6 & 1.36 & 2 & $\begin{array}{lll}4 & 1\end{array}$ & 1.36 & - & \begin{tabular}{l|l}
12 & 2 \\
\end{tabular} & 2.71 & 13 & \begin{tabular}{l|l}
20 & 7.
\end{tabular} & 7.47 & 9 & \begin{tabular}{|l|}
2.70 \\
\end{tabular} & - & 14 & \begin{tabular}{|l|} 
\\
3.17
\end{tabular} & 10 & \begin{tabular}{l|l}
20 & \\
\end{tabular} & 6.79 & - & \begin{tabular}{l|l}
16 & 3
\end{tabular} & 3.62 & - & 71 & 1.58 & - & 10 & 2.26 & - & 6 & 1.36 & -1 & 8 & \begin{tabular}{|l|}
.81 \\
\end{tabular} & 3 & 36 & \begin{tabular}{|l|} 
\\
8.82
\end{tabular} & Dodec \\
\hline cia-Alban & \begin{tabular}{|l|l}
392 \\
392
\end{tabular} & 270 & 189 & \begin{tabular}{l|l}
18 & 76
\end{tabular} & & - & 13 & 4.81 & 1 & - & 0.37 & 2 & 1 & 1.11 & - & $\begin{array}{ll}2 & \end{array}$ & 0.74 & - & \begin{tabular}{l|l}
2 & 0
\end{tabular} & 0.74 & 3 & \begin{tabular}{l|l}
3 & 2.2
\end{tabular} & 2.22 & 7 & 2.59 & - & 5 & 1.85 & 1 & $\begin{array}{ll}7 & 2 \\
7\end{array}$ & 2.96 & - & \begin{tabular}{l|l}
4 & 1
\end{tabular} & 1.48 & 1 & $\begin{array}{ll}2 & 1\end{array}$ & 1.11 & - & 3 & 1.11 & -1 & 1 & 0.37 & -1 & 2 & \begin{tabular}{|l|}
.1 .16 \\
0.74
\end{tabular} & - & 3 & 1.11 & Grecia \\
\hline Crativantas & \begin{tabular}{|l|}
392 \\
391
\end{tabular} & 198 & 94 & $\begin{array}{ll}2 & 48\end{array}$ & 8.49 & 3 & 6 & 4.55 & 2 & 5 & 3.53 & - & 8 & 4.04 & 1 & $\begin{array}{ll}2 & 9 \\
9 & 5\end{array}$ & 5.05 & 1 & \begin{tabular}{l|l}
7 & 4 \\
7
\end{tabular} & 4.04 & 11 & \begin{tabular}{l|l}
6 & 8. \\
6
\end{tabular} & \begin{tabular}{c|c}
.59 & -
\end{tabular} & 10 & 5.05 & - & 8 & \begin{tabular}{|}
$\mid 1.00$ \\
4.04
\end{tabular} & 1 & \begin{tabular}{l|l}
6 & 3 \\
6
\end{tabular} & 3.53 & - & \begin{tabular}{l|l}
5 & 2 \\
5
\end{tabular} & 2.52 & - & \begin{tabular}{l|l}
2 & \\
5 & 2
\end{tabular} & 2.52 & - & 1 & 0.50 & - & & 0.50 & - & 2 & $\begin{array}{l}.04 \\
1.01\end{array}$ & - & 4 & $\left|\begin{array}{|}\mid 1.11 \\
2.02\end{array}\right|$ & \begin{tabular}{|l|} 
Albani \\
\end{tabular} \\
\hline ia Meridionale & 368 & 586 & 362 & $\begin{array}{lll}17 & 64\end{array}$ & 3.68 & & 25 & 4.44 & - & 18 & 3.07 & - & 3 & 0.51 & - & 7 & 1.19 & 1 & \begin{tabular}{l|l}
3 & 0
\end{tabular} & 0.68 & 3 & \begin{tabular}{l|l}
7 & 1. \\
\end{tabular} & \begin{tabular}{l|l}
1.71 & 1 \\
\end{tabular} & 6 & \begin{tabular}{|l}
1.19 \\
\end{tabular} & - & 20 & 3.41 & 10 & \begin{tabular}{l|l}
13 & 3 \\
\end{tabular} & 3.93 & 1 & \begin{tabular}{l|l}
11 & 2
\end{tabular} & 2.05 & - & \begin{tabular}{l|l}
12 & 2
\end{tabular} & 2.05 & - & 13 & 2.22 & - & 5 & 0.85 & - & 9 & 1.54 & 3 & 35 & \begin{tabular}{|l|l|}
6.48 \\
\end{tabular} & Grecia Me \\
\hline slavia & \begin{tabular}{|l}
383 \\
\end{tabular} & 236 & 109 & \begin{tabular}{l|l}
7 & $4 !$
\end{tabular} & 99.15 & 17 & 6 & 9.74 & 4 & 6 & 4.25 & 1 & 5 & 2.54 & 2 & \begin{tabular}{l|l}
4 & 2 \\
4
\end{tabular} & 2.54 & 3 & \begin{tabular}{l|l}
6 & 3 \\
\end{tabular} & 3.81 & 24 & \begin{tabular}{l|l}
7 & 13.1
\end{tabular} & 3.14 & 9 & 4.25 & 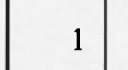 & 4 & 2.13 & - & \begin{tabular}{l|l}
7 & 2 \\
\end{tabular} & 2.97 & - & \begin{tabular}{l|l}
2 & 0
\end{tabular} & 0.84 & 1 & & 1.70 & - & 2 & 0.84 & - & 2 & 0.84 & - & 1 & 0.42 & - & & \begin{tabular}{|l|} 
\\
\end{tabular} & Iugoslavia \\
\hline Egeo & 365 & 777 & 563 & $\begin{array}{ll}32 & 76\end{array}$ & 6.58 & 1 & 32 & 4.25 & 8 & 14 & 2.83 & & 11 & 2.32 & 2 & $\begin{array}{l}9 \\
9\end{array}$ & 1.42 & - & \begin{tabular}{l|l}
8 & 1
\end{tabular} & 1.03 & 47 & \begin{tabular}{l|l}
7 & 6.4
\end{tabular} & 6.95 & 5 & \begin{tabular}{|l|}
1.16 \\
\end{tabular} & 3 & 6 & \begin{tabular}{|l|}
1.16 \\
\end{tabular} & ${ }^{4}$ & \begin{tabular}{l|l}
2 & 0 \\
\end{tabular} & 0.77 & - & \begin{tabular}{l|l}
1 & 0
\end{tabular} & 0.13 & 1 & & 0.25 & - & 3 & 0.37 & -1 & - & - & - & 1 & 0.13 & - & 5 & $|0.64|$ & Mar Egeo \\
\hline & 370 & 470 & 187 & \begin{tabular}{l|l}
10 & 4
\end{tabular} & 11.91 & - & 15 & 3.19 & - & & $\mid 1.28$ & 2 & 10 & 2.55 & - & 811 & 1.70 & - & \begin{tabular}{l|l}
14 & 2 \\
\end{tabular} & 2.98 & 11 & \begin{tabular}{l|l}
21 & 6.
\end{tabular} & 6.81 & 28 & \begin{tabular}{|c|}
6.17 \\
\end{tabular} & 2 & 31 & 7.02 & 4 & \begin{tabular}{l|l}
40 & 9 \\
9
\end{tabular} & 9.36 & - & \begin{tabular}{l|l}
21 & 4 \\
\end{tabular} & 4.47 & - & \begin{tabular}{l|l}
17 & 3 \\
17 &
\end{tabular} & 3.62 & - & 13 & 2.77 & - & & 1.49 & - & 8 & 1.70 & - & 14 & 2.98 & \begin{tabular}{|l|} 
Creta \\
\end{tabular} \\
\hline Mediterraneo Orier & 371 & 127 & 56 & 24. & & - & - & - & - & & \begin{tabular}{l|l}
0.79 \\
\end{tabular} & 1 & & 1.57 & - & 1 & 0.79 & - & \begin{tabular}{l|l}
4 & 3 \\
4
\end{tabular} & 3.15 & 10 & & $2.60 \mid-$ & & 5.51 & - & 7 & 5.51 & - & 6. & 4.72 & - & & 5.51 & - & - & & - & 6 & 4.72 & 1 & & 1.57 & - & 1 & & - & & 7.09 & Medite \\
\hline Stretto Gibillerra & 384 & 474 & 88 & $\left.2\right|_{18}$ & & & & & & & & & & $|1.48|$ & 3 & & 1.051 & 21 & & $0.84 \mid 3$ & & & & & & & & & & & & & & & & & & & & & & & & & & & & & & \\
\hline $\begin{array}{l}\text { Mar Tirreno } \\
\text { Turchia }\end{array}$ & $\begin{array}{l}389 \\
366\end{array}$ & & & & & & & & & & & & & & solo o'té & 'equazior & one ed $\mathrm{i}$ & il grafico & co corris & risponder & lente. (V & (Vedi Ta & Fabella 2b) & & & & & & & & & & & & & & & & & & & & & & & & & & & \\
\hline
\end{tabular}


moti). Anche il basso Tirreno presenta una scarsa attività (solo 25 terremoti) però esso costituisce un quadro tutto particolare;

2) quanto alla profondità: quasi ovunque il numero dei terremoti con ipocentro prossimo allo zero è elevatissimo. La Grecia conta addirittura 996 terremoti superficiali (pari a circa il $73 \%$ di tutti i suoi eventi sismici) e 645 ne conta la Turchia (per $h=0$ ). In quasi tutte le regioni la maggior parte dei terremoti proviene da profondità minori di $50 \mathrm{~km}$ con un generale accumulo, come si è visto, a $33 \mathrm{~km}$.

TABELLA $1 b$

Mar Tirreno

\begin{tabular}{|c|c|c|}
\hline \multicolumn{2}{|c|}{ N. totale di terremoti: 25 (tutti con errore) } \\
\hline$h(\mathrm{~km})$ & $\begin{array}{c}\text { numero di terremoti } \\
\text { per ogni intervallo di } \\
\text { profondità }\end{array}$ & $\bar{\varepsilon}_{i}$ \\
\hline 0 & 1 & - \\
53 & 1 & 54.72 \\
$100 \div 200$ & 2 & 18.10 \\
$200 \div 250$ & 3 & 1.40 \\
$250 \div 300$ & 9 & 1.25 \\
$300 \div 350$ & 5 & 1.32 \\
$350 \div 400$ & 1 & 0.69 \\
$400 \div 500$ & 3 & 1.53 \\
\hline
\end{tabular}

Solo Grecia, Turchia e Dodecanneso hanno una sensibile attività proveniente da profondità superiori ai $75 \mathrm{~km}$; ma le punte più profonde spettano indubbiamente al basso Tirreno, con terremoti che per tre volte hanno raggiunto profondità comprese tra 400 e $500 \mathrm{~km}$. 
Gli errori associati al calcolo di h e la loro entità - Andamento degli errori in funzione della profondità ipocentrale - Calcolo delle curve $\bar{\varepsilon}=\beta h^{-\alpha}$ (per ogni regione)

In questa fase della ricerca trova conferma quanto già rilevato nella sommaria indagine mondiale di cui si è parlato nel Cap. 1. In ciascuna regione quasi la totalità dei terremoti posti alle due profondità $h=0, h=33 \mathrm{~km}$ sono privi di errore e quasi ovunque il loro numero è molto elevato, specialmente $h=0$. Per quanto riguarda le altre profondità resta convalidato, anche in questa indagine molto più documentata della generale, il fatto che gli errori sono assai più rilevanti per $i$ terremoti crostali che non per gli altri, alcuni addirittura assurdi! Solo alcuni esempi scelti fra tanti:

$\begin{array}{llll}8 \text { settembre } & 1964 & \text { - Italia Settentrionale } & h=10 \pm 50 \mathrm{~km} \\ 5 \text { settembre } & 1968 & \text { - Dodecanneso } & h=1 \pm 44 \mathrm{~km} \\ 31 \text { gennaio } & 1970 & \text { - Dodecanneso } & h=6+117 \mathrm{~km} \\ 17 \text { luglio } & 1972 & \text { - Mar Jonio } & h=16+80 \mathrm{~km}\end{array}$

Nel tentativo di dare un aspetto matematico al problema si è concentrata l'attenzione sulle sole profondità corredate degli errori corrispondenti. Pertanto in ciascuna regione, per ogni classe di $h_{\mathrm{i}}$ è stato calcolato l'errore relativo percentuale [E] mediato sugli $n$ eventi ivi avvenuti:

$$
\bar{\varepsilon}_{i}=\frac{1}{n} \sum\left(\frac{\delta h_{\mathrm{i}}}{h_{\mathrm{i}}}\right) \%
$$

I valori numerici ottenuti (vedi tabella $2 \mathrm{a}$ ) sono stati riportati nella serie dei punti delle figg. $2(2 / 1 \ldots 2 / 19)$. Questi punti si distribuiscono con un andamento medio generale che si ripete con varianti più o meno accentuate in tutte le regioni esaminate; 
TABELLA $2 a$

ERrore RElativo PERCENTUALE MEdio PER OGNI Classe di PROFONDITA' (PER OGNi REGIONE)

\begin{tabular}{|c|c|c|c|c|c|c|c|c|c|c|c|c|c|c|c|c|c|c|}
\hline $\begin{array}{c}\text { Regioni } \\
h \\
(\mathrm{~km})\end{array}$ & $\begin{array}{c}\text { Italia } \\
\text { Sett. } \\
(545) \\
\end{array}$ & $\begin{array}{c}\text { Italia } \\
\text { Centr. } \\
(381) \\
\end{array}$ & $\begin{array}{c}\text { Italia } \\
\text { Merid. } \\
(390) \\
\end{array}$ & $\begin{array}{c}\text { Sicilia } \\
(398) \\
\end{array}$ & $\begin{array}{c}\text { Mare } \\
\text { Adriat. } \\
(382) \\
\end{array}$ & $\begin{array}{c}\text { Mare } \\
\text { Ionio } \\
(399) \\
\end{array}$ & $\begin{array}{c}\text { Algeria } \\
(396) \\
\end{array}$ & $\begin{array}{c}\text { Grecia } \\
(364) \\
\end{array}$ & $\begin{array}{c}\text { Dode- } \\
\text { canneso } \\
(369) \\
\end{array}$ & $\begin{array}{c}\text { Grecia- } \\
\text { Albania } \\
(392) \\
\end{array}$ & $\begin{array}{c}\text { Albania } \\
(391) \\
\end{array}$ & $\begin{array}{c}\text { Grecia } \\
\text { Merid. } \\
(368) \\
\end{array}$ & $\begin{array}{c}\text { Iugo- } \\
\text { slavia } \\
(383) \\
\end{array}$ & $\begin{array}{l}\text { Mare } \\
\text { Egeo } \\
(365) \\
\end{array}$ & $\begin{array}{l}\text { Creta } \\
(370) \\
\end{array}$ & $\begin{array}{c}\text { Medit. } \\
\text { Orient. } \\
(371) \\
\end{array}$ & $\begin{array}{c}\text { Gibil- } \\
\text { terra } \\
(384) \\
\end{array}$ & $\begin{array}{c}h \\
(\mathrm{~km}) \\
\end{array}$ \\
\hline 2.5 & 578 & 464.8 & 300 & $(h=2.5) 690$ & 737.5 & 737 & 750 & 356 & 370 & 251 & 512 & 365 & 347 & - & 399 & 513.5 & 117.5 & 2.5 \\
\hline 7.5 & 177.8 & 181.8 & - & & - & 138 & 157 & 121 & 237.7 & 108 & 173 & 107.3 & 154 & 237 & 247 & - & 215 & 7.5 \\
\hline 12.5 & 154 & 73.7 & 130 & $(h=15) 191$ & 55 & 75 & 108 & 103 & 151.6 & - & 98.2 & 108.1 & 92 & 113 & 90.6 & 158 & 78 & 12.5 \\
\hline 17.5 & 193.3 & 115.7 & 88.7 & & - & (335) ? & 94 & 113 & 77.6 & 87 & 68.6 & 102.6 & 99 & 159 & 62 & 215 & 105 & 17.5 \\
\hline 22.5 & 66.3 & 73.0 & 73.45 & $(h=23 \quad) 76.3$ & 70.5 & 93 & 87 & 56.5 & 46.2 & 121 & 55.7 & 80.42 & 58 & 133 & 84 & 68 & 34.5 & 22.5 \\
\hline 27.5 & 63.2 & 63.0 & - & & - & 76 & 28 & 62.2 & 59.5 & 65 & 53 & 72.3 & 50 & 64 & 80 & 60.5 & 43 & 27.5 \\
\hline 32.5 & 39.3 & 167.0 & 15.42 & $(h=33 \quad 43$ & - & 69 & 44 & 28.7 & 35.5 & 45 & 29 & 41.1 & 51 & 31.6 & 26.5 & 26.3 & - & 32.5 \\
\hline 37.5 & 20.3 & 25.5 & 36.08 & $(h=37 \quad) 21$ & 39 & 32 & 30 & 26.1 & 28.5 & 31 & 26 & 33.16 & 30 & 34.4 & 23.8 & 28.8 & 49 & 37.5 \\
\hline 42.5 & 26.6 & 53.8 & 14.10 & & 15 & 24 & - & 23.4 & 25.8 & 19 & 24 & 23.3 & 45 & 30 & 25.1 & 33.8 & 27.6 & 42.5 \\
\hline 47.5 & 29.8 & 35.0 & 16.30 & & - & 33 & - & 25 & 23.4 & 22 & 21 & 14.25 & 43 & 30 & 20 & 30.3 & 16 & 47.5 \\
\hline 52.5 & 22 & - & 24.60 & & 57.4 & 27 & 26.5 & 19 & 15.9 & 18 & 28.6 & 21.10 & 32 & 20 & 20 & 18 & 24 & 52.5 \\
\hline 57.5 & 34.3 & 26.5 & - & $(h=58 \quad) 33$ & 60.3 & 37.2 & 36 & 22.8 & 23.7 & 16 & 18.6 & 22.50 & 39 & 30 & 17 & - & 13.8 & 57.5 \\
\hline 62.5 & 29.5 & - & - & & 18.0 & 16 & & 14.4 & 20 & - & 23 & 21.60 & 21 & 44.3 & 17 & 11.93 & - & 62.5 \\
\hline 67.5 & 4.3 & 97.5 & 14.5 & & & 25.5 & & 36.7 & 14.1 & 14.5 & 7.5 & 14.50 & 23 & - & 12 & 61 & 17.5 & 67.5 \\
\hline 72.5 & - & 53.5 & - & & & - & & 16.1 & 24.8 & 21.0 & 24 & 12.70 & 26 & 67 & 13.4 & 57 & - & 72.5 \\
\hline 77.5 & - & & 12.6 & & & 45 & & 10.2 & 7.2 & & 13 & 8.90 & - & - & 19 & 25.5 & - & 77.5 \\
\hline 82.5 & 28.5 & & 79.7 & & & & & 12.8 & 10.1 & & 35 & 19.50 & - & 35 & 5.97 & 28 & - & 82.5 \\
\hline 87.5 & & & - & & & & & 12.0 & 4.1 & & 24.5 & 8.70 & - & & 6.86 & 21.5 & 7.2 & 87.5 \\
\hline 92.5 & & & 21.5 & & & & & 12.0 & 60 & & & - & & & 8.10 & 26.3 & 19 & 92.5 \\
\hline 97.5 & & & & & & & & & 36.4 & & & 9.60 & & & 13 & & - & 97.5 \\
\hline 102.5 & & & & & & & & & 8.1 & & & 5.7 & & & & & 12 & 102.5 \\
\hline 107.5 & & & & & & & & & & & & & & & & & - & 107.5 \\
\hline
\end{tabular}


tale andamento si può ritenere, nel suo complesso, vagamente esponenziale.

Abbiamo quindi tentato di definire analiticamente la funzione che descrive tale andamento ponendo che essa sia del tipo

$$
\bar{\varepsilon}_{i}=\beta h_{1}-\alpha
$$

Poiché dalla [1] si trae l'equazione lineare (a termini logaritmici)

$$
\log \bar{\varepsilon}_{i}=-\alpha \log h_{\mathbf{i}}+\log \beta
$$

per ogni regione geografica esaminata abbiamo risolto il sistema di equazioni lineari [2] nel piano

$$
\begin{aligned}
& \mathrm{X}=\log h \\
& \mathrm{Y}=\log \bar{\varepsilon}
\end{aligned}
$$

per poterne calcolare $i$ termini noti $« \log \beta$ » e i coefficienti angolari « $\alpha$ » con il metodo dei minimi quadrati. I risultati sono riportati nella tabella $2 b$ (ivi è indicato anche il numero dei punti usati per il calcolo delle [2]).

Le relazioni trovate sono nella quasi totalità dei casi abbastanza ben definite, soprattutto per le regioni più sismicamente attive per le quali si è potuto utilizzare un maggior numero di dati. (Na questa indagine si è escluso il Mar Tirreno, sede di eventi molto profondi, per il quale l'andamento degli errori è 
TABELLA $2 b$

\begin{tabular}{|c|c|c|c|c|c|}
\hline Regioni & n. & $\begin{array}{c}\text { Num. } \\
\text { dei } \\
\text { punti } \\
\text { usati }\end{array}$ & $\log \bar{E}=\alpha \log h+\log \beta$ & $\bar{\varepsilon}=\beta h^{-\alpha}$ & $\begin{array}{c}\text { Reg. } \\
\text { n. }\end{array}$ \\
\hline Italia Settentr. & $(545)$ & 15 & $\log \bar{\varepsilon}=-1.1159 \log h+3.3125$ & $\bar{\varepsilon}=2053.5250 r^{-1.1159}$ & (545) \\
\hline Italia Centrale & $(381)$ & 13 & $\log \bar{\varepsilon}=-0.6554 \log h+2.8094$ & $\bar{\varepsilon}=644.7628 h-0.6554$ & $(381)$ \\
\hline Italia Merid. & $(390)$ & 13 & $\log \bar{\varepsilon}=-0.8196 \log h+2.8176$ & $\bar{\varepsilon}=657.0524 h^{-0.8196}$ & $(390)$ \\
\hline Sicilia & $(398)$ & 6 & $\log \bar{\varepsilon}=-1.1052 \log h+3.3401$ & $\bar{\varepsilon}=2188.2654 h^{-1.1052}$ & $(398)$ \\
\hline Mar Adriatico & $(382)$ & 8 & $\log \bar{\varepsilon}=-0.9334 \log h+3.0736$ & $\bar{\varepsilon}=1184.6771 h^{-0.9334}$ & $(382)$ \\
\hline Mar Jonio & $(399)$ & 14 & $\log \bar{\varepsilon}=-0.9188 \log h+3.0798$ & $\bar{\varepsilon}=1201.7109 h^{-0.9188}$ & $(399)$ \\
\hline Algeria & $(396)$ & 10 & $\log \bar{\varepsilon}=-1.0375 \log h+3.2021$ & $\bar{\varepsilon}=1592.5754 h^{-1.0375}$ & $(396)$ \\
\hline Grecia & $(364)$ & 19 & $\log \bar{\varepsilon}=-0.9824 \log h+3.0360$ & $\bar{\varepsilon}=1086.4256 h^{-0.9824}$ & $(364)$ \\
\hline Dodecanneso & $(369)$ & 21 & $\log \underline{\varepsilon}=-1.0183 \log h+3.1176$ & $\bar{\varepsilon}=1310.9919 h^{-1.0183}$ & $(369)$ \\
\hline Grecia-Albania & $(392)$ & 13 & $\log \bar{\varepsilon}=-0.8838 \log h+2.8963$ & $\bar{\varepsilon}=787.5896 h^{-0.8838}$ & (392) \\
\hline Albania & $(391)$ & 18 & $\log \bar{\varepsilon}=-0.9652 \log h+3.0420$ & $\bar{\varepsilon}=1101.5393 h^{-0.9652}$ & (391) \\
\hline Grecia Merid. & $(368)$ & 20 & $\log \varepsilon=-1.0772 \log h+3.1596$ & $\bar{\varepsilon}=1444.1091 h^{-1.0772}$ & $(368)$ \\
\hline Jugoslavia & $(383)$ & 15 & $\log \varepsilon=-0.7930 \log h+2.8715$ & $\overline{\mathbf{E}}=743.8751 h^{-0.7930}$ & $(383)$ \\
\hline Mar Egeo & $(365)$ & 14 & $\log \bar{\varepsilon}=-0.8763 \log h+3.0816$ & $\underline{\bar{\varepsilon}}=1206.7019 h^{-0.8763}$ & $(365)$ \\
\hline Creta & $(370)$ & 20 & $\log \bar{\varepsilon}=-1.1515 \log h+3.2591$ & $\underline{\bar{\varepsilon}}=1815.9337 h^{-1.1515}$ & $(370)$ \\
\hline Mediterr. Orien. & $(371)$ & 17 & $\log \underline{\bar{\varepsilon}}=-0.9007 \log h+3.0822$ & $\bar{\varepsilon}=1208.3702 h^{-0.9007}$ & (371) \\
\hline Gibilterra & $(384)$ & 15 & $\log \bar{\varepsilon}=-0.8209 \log h+2.7520$ & $\bar{\varepsilon}=564.9370 h^{-0.8209}$ & (384) \\
\hline Turchia & $(366)$ & 95 & $\log \bar{\varepsilon}=-0.96 \quad \log h+3.13$ & $\bar{\varepsilon}=1348.96$ & (366) \\
\hline
\end{tabular}


del tutto diverso: si rimanda alla tabella la per i valori numerici). Dalle [2], noti $« \alpha » \mathrm{e} \| \log \beta »$ si risale immediatamente alla forma esponenziale [1].

Le equazioni corrispondenti alle singole regioni sono anche esse riportate nella tabella $2 \mathrm{~b}$ : la serie delle figg. $1 / 1 \ldots$ le rappresenta graficamente.

Le curve trovate sembrano dare una risposta a molti interrogativi. Anche se in linea di massima esse si possono ritenere soddisfacenti, occorre però fare moltissime riserve, in quanto i valori singoli degli errori percentuali medi delle $h$ si discostano notevolmente dalla media rappresentata dalle equazioni.

Nel prossimo paragrafo analizzeremo accuratamente i risultati ottenuti.

ANALISI DEI RISULTATI

\section{Un tentativo di interpretazione}

Si è accennato nel paragrafo precedente come l'aver trovato dei valori medi accettabili non risolva però il problema in maniera soddisfacente: infatti permangono sempre dei grossi scarti che fanno nascere ragionevoli dubbi.

In molte delle regioni esaminate gli errori percentuali trovati per le profondità comprese tra 10 e $20 \div 25 \mathrm{~km}$, si discostano sensibilmente dall'andamento medio delle curve calcolate (vedi ad esempio i grafici 2 relativi alla Grecia, alla Grecia Meridionale, al Mar Egeo, al Mediterraneo Orientale e altre).

Qualora questi scarti non fossero casuali, la loro presenza potrebbe suggerire un tentativo di intepretarli come elementi di denuncia di eventuali strati di discontinuità. In tal caso verrebbe sottolineato il fatto che usare per i calcoli un unico tipo di dromocrona può diventare non corretto qualora intervenga un cambiamento nella velocità di propagazione delle prime onde sismiche che sono quelle usate per il calcolo dei dati ipocentrali.

A questo proposito vogliamo richiamare certi risultati che mettono in chiara luce questa osservazione. 
In uno studio condotto sui terremoti del Friuli del maggio 1976 (Console, Gasparini, 1976), sono stati trovati valori differenti di $h$ a seconda del modello di dromocrona usata, e precisamente:

(FriULI, 1976)

VALORI DI $h$ A SECONDA DEI MODELLI USATI

\begin{tabular}{|c|c|c|c|c|}
\hline $\begin{array}{c}\text { modello } \\
\text { Di Fil.-Marc. }\end{array}$ & $\begin{array}{c}\text { modello } \\
\text { Caloi }\end{array}$ & $\begin{array}{l}\text { modello } \\
\text { Hypo } 71\end{array}$ & $\begin{array}{l}\text { modello } \\
\text { Appennini }\end{array}$ & $\begin{array}{c}\text { modello } \\
\text { Alpi }\end{array}$ \\
\hline $40.763 \pm 2.301$ & $23.894 \pm 2.629$ & $12.248 \pm 2.504$ & $18.256 \pm 2.466$ & $21.829 \pm 3.120$ \\
\hline $33.435+3.425$ & $20.088+3.797$ & $1.231 \pm 5.570$ & $8.194 \pm 3.205$ & $9.995 \pm 0.014$ \\
\hline
\end{tabular}

Come si vede nell'esempio riportato i calcoli matematici hanno condotto ad errori di $h$ molto contenuti, però è da notare che la stessa scossa si presenta con $h$ estremamente variabile (da 12 a $40 \mathrm{~km}$ e da 1 a $33 \mathrm{~km}$ ) a seconda del modello usato.

Una ulteriore analisi porterebbe ad evidenziare anche il fatto che il numero e la scelta delle stazioni adoperate per il calcolo di $h$ ne influenzano notevolmente il risultato, per cui lo stesso terremoto presenta, ai calcoli valori di $h$ differenti anche a seconda di come viene trattato il problema. La conseguenza logica è che intorno al valore di $h$ prescelto si distribuisce una serie di errori più o meno grandi.

A nostro avviso voler ottimizzare il risultato di $h$ diventa a questo punto solo un virtuosismo matematico.

A prescindere da qualunque altra considerazione risulta 
evidente il fatto che voler rappresentare dei fenomeni naturali con modelli matematici può condurre spesso a valutazioni errate o quanto meno lontane dalla realtà obbiettiva.

Le relazioni trovate nell'analisi condotta in questa nota

$$
\bar{\varepsilon}=f(h)
$$

fanno risaltare, come si è visto, le enormi difficoltà di valutazione che si incontrano specie per terremoti con ipocentro entro la crosta o alla base di essa.

Le anomalie sono tanto meno importanti quanto più i terremoti sono profondi talché si potrebbe desumere che i risultati ottenuti trovano una loro giustificazione nelle ipotesi fatte da vari autori sulla costituzione interna della Terra specie per quanto riguarda il Mantello inferiore e il Mantello superiore (ipotesi che sono servite anche alla costruzione delle dromocrone delle onde sismiche): però non altrettanto si può dire per la crosta dove le equazioni trovate conducono ad errori percentuali elevatissimi: ma qui, oltre le indiscutibili e non sempre conosciute eterogeneità crostali, può giocare un ruolo importante il fatto che, come si è osservato in precedenza, il focolaio sismico va considerato più come "un volume " che non come « un punto matematico " per cui all'interno di questo volume il valore reale di $h$ può assumere valori differenti: questa differenza, anche se piccola, può contribuire in modo sensibile a variare anche di molto il valore dell' $\varepsilon$ medio.

Cap. 3 - Il problema relatvo alla sola Italia

\section{(Nord-Centro-Sud-Sicilia)}

L'analisi condotta nell'area Mediterranea ha confermato, precisandoli meglio, i punti salienti che erano emersi nel primo sondaggio fatto su scala mondiale. 
A questo punto, per rendere il problema ancor più idoneo ad uno studio regionale abbiamo ritenuto opportuno restringere ulteriormente l'area di ricerca e, come si era preannunziato, abbiamo preso in considerazione la sola regione italiana, unificandone però le sezioni principali per avere una indicazione mediata sull'intero territorio nazionale, anche in vista del fatto che le singole suddivisioni geografiche proposte dagli autori americani non sembrano rispondere a particolari criteri geofisici di suddivisione. Abbiamo considerato pertanto le sezioni contrassegnate come

\author{
Italia Settentrionale (545) \\ Italia Centrale \\ Italia Meridionale \\ Sicilia

escludendo da questo raggruppamento il Mar Adriatico, il Mar Jonio, e il Mar Tirreno.

La tabella 3 compendia il risultato di questa unificazione e l'equazione che se ne trae è la seguente:

$$
\log \bar{\varepsilon}=-0.7881 \log h+2.9543
$$

o l'equivalente

$$
\bar{\varepsilon}=900.1191 h^{-0.7881}
$$

Il grafico di fig. 3 rappresenta la (a'). 
TABELLA 3

\begin{tabular}{|cc|cc|}
\hline$h(\mathrm{~km})$ & $\bar{\varepsilon}$ & $h(\mathrm{~km})$ & $\bar{\varepsilon}$ \\
\cline { 3 - 4 } 2.5 & 470.82 & 47.5 & 29.44 \\
7.5 & 179.28 & 52.5 & 23.75 \\
12.5 & 106.00 & 57.5 & 40.21 \\
17.5 & 135.60 & 62.5 & - \\
22.5 & 91.13 & 67.5 & 70.00 \\
27.5 & 63.17 & 72.5 & 53.52 \\
32.5 & 60.08 & 77.5 & 12.65 \\
37.5 & 32.55 & 82.5 & 45.33 \\
42.5 & 41.60 & & \\
\hline
\end{tabular}

Errori percentuali medi alle varie profondità per il solo territorio italiano (Italia Settentrionale - Centrale - Meridionale - Sicilia).

DisCUSSIONE DEI RISULTATI RELATIVI AL SOLO TERRITORIO NAZIONALE

Anche qui l'esame del grafico 3 rappresentativo della equazione (a') offre lo spunto alle seguenti considerazioni:

- per $h<10 \mathrm{~km}$ gli errori percentuali medi sono elevatissimi, al di fuori di ogni logica interpretazione;

- nell'intervallo $10 \div 20 \mathrm{~km}$ si nota una forte dispersione intorno alla curva mediata;

- nella parte inferiore della crosta, tra 20 e $35 \mathrm{~km}$, gli errori si adattano abbastanza bene alla curva pur mantenendosi superiori al 50\%;

- per $h$ compreso tra 35 e $60 \mathrm{~km}$ (Mantello superiore) essi migliorano sensibilmente non superando il $40 \%$;

- invece una nuova dispersione si presenta a profondità superiori ai $65 \mathrm{~km}$. 


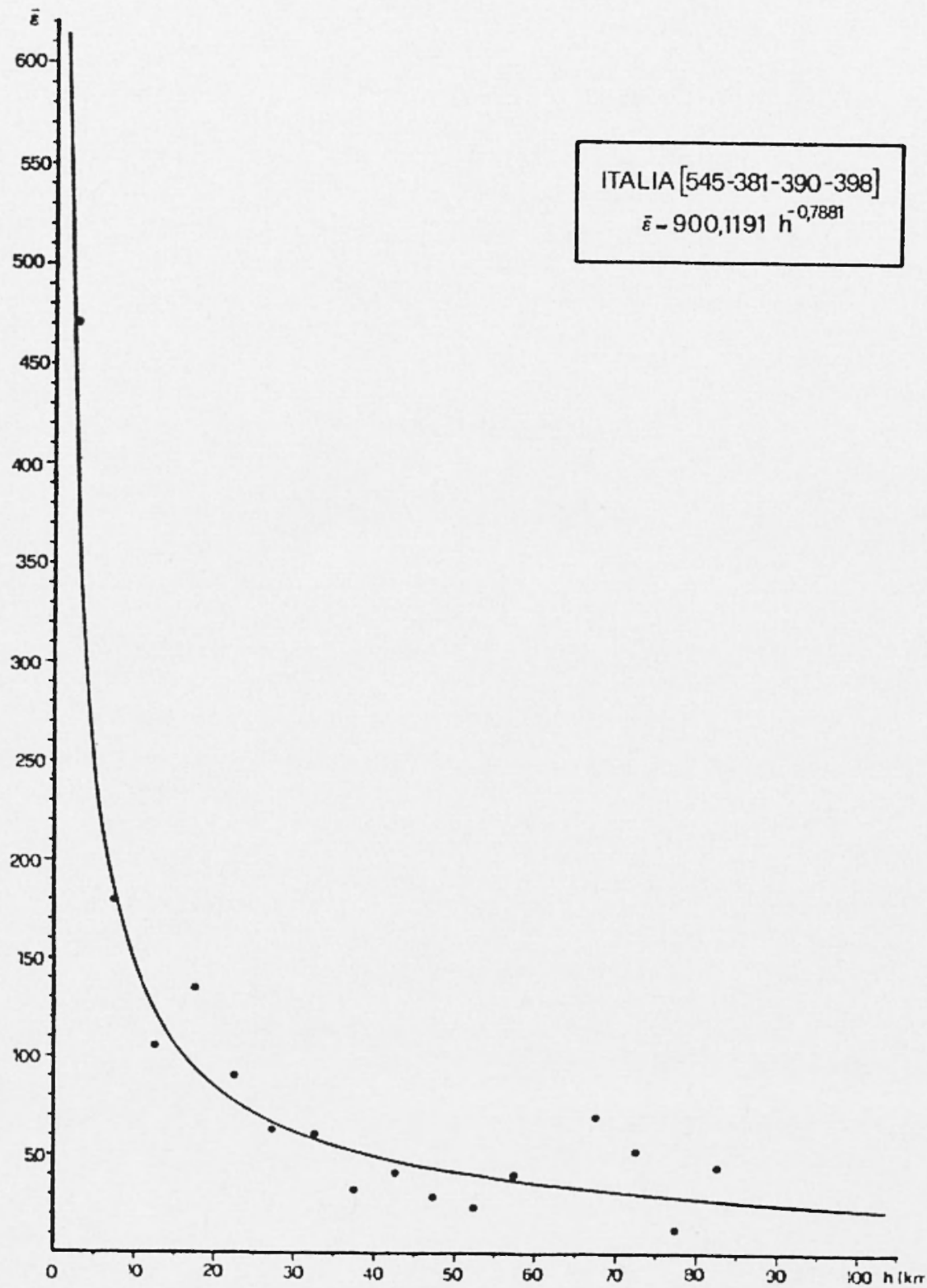

Fig. 3 - Curva $\varepsilon=\beta h^{-\alpha}$ (vale per l'Italia unificata: Nord. Centro-Sud-Sicilia). 
Le osservazioni precedenti potrebbero assumere un interesse particolare se si esaminano in parallelo i modelli crostali delle dromocrone sperimentali in uso per l'Italia e i modelli crostali delle dromocrone sintetiche. Ma questo significherebbe voler dare alla ricerca qui condotta una interpretazione che va al di là delle nostre intenzioni.

Preferiamo pertanto non formulare alcuna ipotesi al riguardo e lasciare il campo aperto ad ulteriori indagini.

CaP. 4 - Sintesi di carattere generale

\section{Conclusioni}

Abbiamo preferito piuttosto riassumere nella tabella 4 e nella Fig. 4 ad essa associata, gli elementi che abbiamo ritenuto più significativi ai fini della nostra analisi critica.

Supposto, per convenzione generale, lo spessore medio della crosta terrestre di $33 \mathrm{~km}$ abbiamo considerato tre intervalli di profondità

$$
h<10 \mathrm{Km} \quad 10<h<20 \mathrm{Km} \quad 20<h<30 \mathrm{Km}
$$

per ciascuno dei quali abbiamo riportato i corrispondenti intervalli di variazione degli $\bar{\varepsilon}$

Nel primo intervallo ( $h<10 \mathrm{~km}$ ) come si è visto, in tutte le regioni esaminate l'errore percentuale medio è elevatissimo e, ripetiamo, al di fuori di ogni significato fisico.

Anche nel secondo intervallo $(10 \leq h<20)$ gli errori si mantengono molto elevati, tutti superiori al $50 \%$ ad eccezione dello Stretto di Gibilterra che scende al valore minimo del $48 \%$.

Invece nel terzo intervallo $(20 \leq h \leq 30)$ la maggior parte degli $\bar{\varepsilon}$ comincia a scendere al di sotto del $50 \%$.

Nella penultima colonna della tabella 4 sono stati riportati 
gli errori percentuali medi calcolati alla base della Moho supposta convenzionalmente a $33 \mathrm{~km}$. A questa profondità in generale gli errori scendono al di sotto del $50 \%$.

Restano ancora elevati nell'Italia Centrale (65.19\%), nel Mar Egeo (56.35\%), nel Mediterraneo Orientale (51.82\%) e nell'Italia comprensiva delle 4 sezioni principali $(57.2 \%)$.

Ci è parso interessante evidenziare, per ogni regione, anche il valore di $h$ oltre cui l'errore associato alla profondità diventa minore del $50 \%$. Vista l'enorme difficoltà che si incontra nel calcolo di $h$ e vista la grande incertezza che accompagna quasi sempre il dato di profondità calcolato, può sembrare ragionevole anche se molto discutibile ritenere accettabili errori di valutazione inferiori al $50 \%$, finché ci si mantiene all'interno della crosta.

L'ultima colonna della tabella 4 riporta pertanto questi dati di profondità limite.

La Fig. 4 consente una immediata visione di questa sintesi.

A termine dell'analisi critica condotta nel presente lavoro, pur rifiutandoci di azzardare qualunque ipotesi interpretativa ci sembra di poter concludere dicendo che tra i dati ipocentrali di un terremoto, quello che dà adito a molti dubbi è il dato di profondità, tanto più incerto quanto più prossimo alla superficie esso si trova.

La scelta della dromocrone usate gioca un ruolo molto importante. Converrebbe forse meglio condurre i calcoli sul rilevamento sperimentale delle onde $p P$ che, appunto perché individuate sul sismogramma, denunciano l'effettiva testimonianza del punto di provenienza dell'onda sismica.

Qualora si facesse uso di dromocrone appropriate la conoscenza degli errori associati al dato di profondità potrebbe fornire elementi utili per una indagine sulla ricerca di eventuali superfici di discontinuità. 
TABELLA 4

ANALISI DEgli ERRori PERCENTUALI MEdJ

(in base alle curve calcolate)

\begin{tabular}{|c|c|c|c|c|c|c|c|c|}
\hline \multirow{4}{*}{\multicolumn{2}{|c|}{ Regioni }} & \multicolumn{5}{|c|}{ crosta } & \multirow{4}{*}{$\begin{array}{c}\bar{\varepsilon} \text { per } \\
\downarrow \\
h=33 \\
\text { (Moho) }\end{array}$} & \multirow[b]{2}{*}{$(*)$} \\
\hline & & \multirow{2}{*}{$\begin{array}{c}\bar{\varepsilon} \text { per } \\
\downarrow \\
h<10 \mathrm{~km}\end{array}$} & \multicolumn{2}{|c|}{$\begin{array}{c}\bar{\varepsilon} \text { per } \\
\vdots \\
\downarrow\end{array}$} & $\begin{aligned} \bar{\varepsilon} & p \\
\downarrow & \end{aligned}$ & & & \\
\hline & & & \multicolumn{2}{|c|}{$10 \leq h<20$} & \multicolumn{2}{|c|}{$20<h<30$} & & \multirow{2}{*}{$\begin{array}{c}h(\mathrm{~km}) \\
\text { (limite) }\end{array}$} \\
\hline & & più di & $\mathrm{da}$ & $\mathrm{a}$ & $\mathrm{da}$ & $\mathrm{a}$ & & \\
\hline (545) & Italia Sett. & 157.3 & 157 & 73 & 73 & 46 & 41.49 & $h=27.92$ \\
\hline (381) & Italia Centr. & 142.6 & 143 & 90 & 90 & 69 & 65.19 & $h=49.46$ \\
\hline (390) & Italia Merid. & 99.5 & 99 & 56 & 56 & 40 & 37.41 & $h=23.17$ \\
\hline$(398)$ & Sicilia & 171.8 & 172 & 80 & 80 & 51 & 45.90 & $h=30.54$ \\
\hline (382) & Mar Adriatico & 138.1 & 138 & 72 & 72 & 49 & 45.31 & $h=29.70$ \\
\hline$(399)$ & Mar Jonio & 144.9 & 145 & 77 & 77 & 53 & 48.37 & $h=31.83$ \\
\hline (396) & Algeria & 146.1 & 146 & 71 & 71 & 47 & 42.33 & $h=28.11$ \\
\hline (364) & Grecia & 113.1 & 113 & 57 & 57 & 38 & 35.01 & $h=22.96$ \\
\hline (369) & Dodecanneso & 125.7 & 126 & 62 & 62 & 41 & 37.26 & $h=24.72$ \\
\hline (392) & Grecia-Albania & 102.9 & 103 & 56 & 56 & 39 & 35.83 & $h=22.63$ \\
\hline$(391)$ & Albania & 119.3 & 119 & 61 & 61 & 41 & 37.70 & $h=24.63$ \\
\hline (368) & Grecia Merid. & 120.9 & 121 & 57 & 57 & 37 & 33.41 & $h=22.70$ \\
\hline (383) & Jugoslavia & 119.8 & 120 & 69 & 69 & 50 & 46.49 & $h=30.10$ \\
\hline (365) & Mar Egeo & 160.4 & 160 & 87 & 87 & 61 & 56.35 & $h=37.83$ \\
\hline (370) & Creta & 128.1 & 128 & 58 & 58 & 36 & 32.40 & $h_{l}=2264$ \\
\hline (371) & Medit. Orien. & 151.9 & 152 & 81 & 81 & 56 & 51.82 & $h=34.33$ \\
\hline (384) & Gibilt. (Str.) & 85.3 & 85 & 48 & 48 & 35 & 32.02 & $h=19.18$ \\
\hline$(366)$ & Turchia & 147.9 & 148 & 76 & 76 & 52 & 47.01 & $h=30.95$ \\
\hline & $\begin{array}{l}\text { Italia } \\
\text { Nord-Centro } \\
\text { Sud-Sic.) }\end{array}$ & 146.6 & 147 & 85 & 85 & 62 & 57.2 & $h=39.16$ \\
\hline
\end{tabular}

$\left.{ }^{*}\right)$ Valori di $h$ oltre cui l'errore diventa minore del $50 \%$. 


\section{BIBLIOGRAFIA}

Bullen K.E., 1975 - The Earth's Density. London.

Console R., Gasparini C. - Analisi dei parametri ipocentrali del terremoto del Friuli (maggio 1976). "Annali di Geofisica", XXIX, 3.

Flinn E.A., Enginhl E.R. Hill A.R. 1974 - Seismic and Geographical Regionalization "Bulletin of the Scismological Society of America ", june.

I.S.C. 1969-1974 - International Seismological Centre, Newbury RG 13 1LA, Berskshire, United Kingdom.

Marcelli L., Pannocchia G., 1974 - Proposta per una classificazione dei dati sperimentali in Sismologia. "Annali di Geofisica", XXVII. 

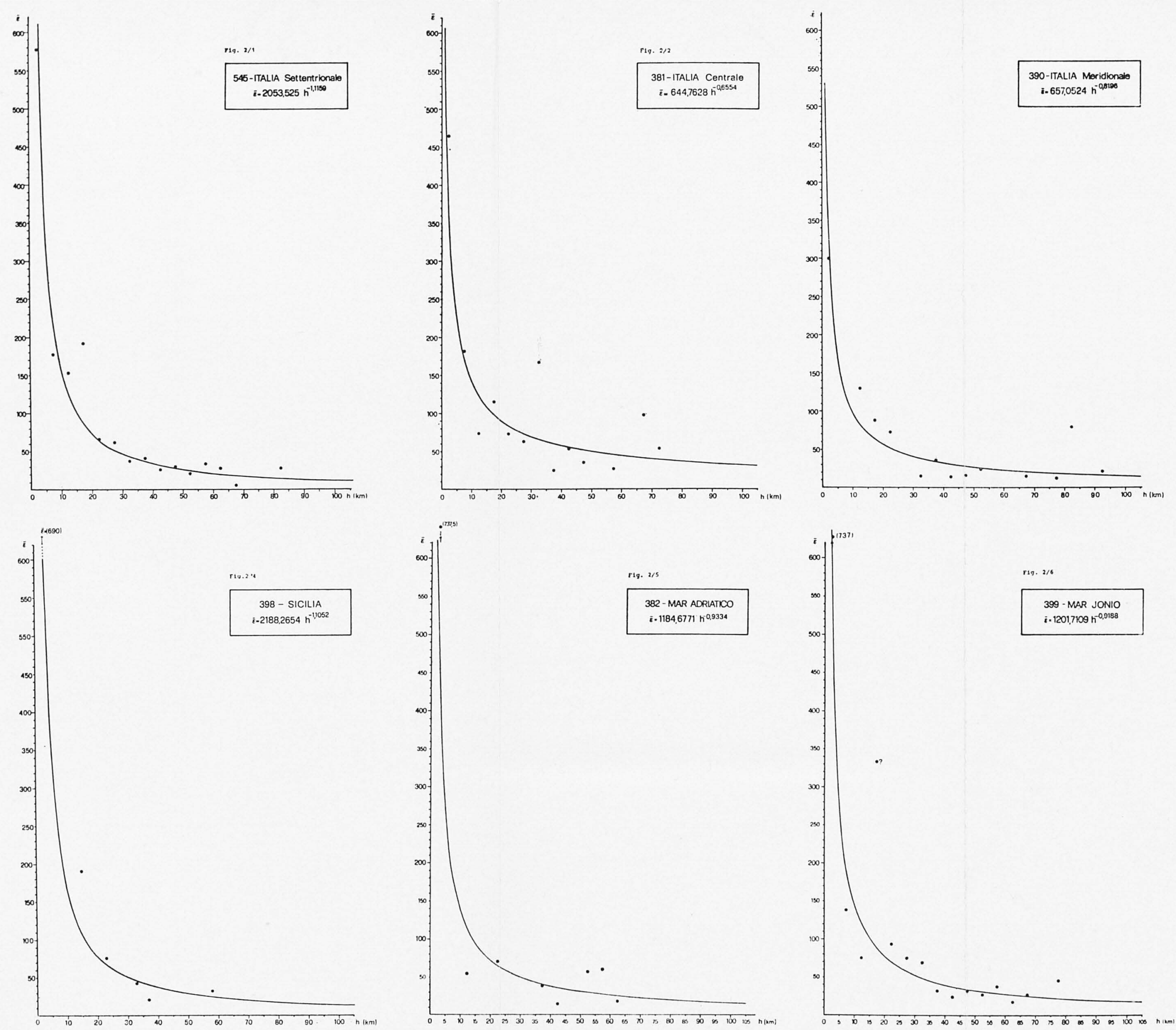

Figg. $2 / 1 \ldots 2 / 19$ - Serie dei grafici rappresentativi delle equazioni $\bar{\varepsilon}=\beta h^{-\alpha}$ per tutte le regioni considerate (con esclusione del Mar Tirreno). Curve $\varepsilon=f(h)$ (Andamento degli errori e serie dei grafici [vale per la tabella 2a (punti) e per la tabella $2 b$ (curve)] 
- indica $i l$ limite inferiore di $\bar{\varepsilon}$ per $\mathrm{h}<10 \mathrm{~km}$

$\Delta \div \mathrm{X}$ indica l'intervallo di variazione di $\bar{\varepsilon}$ per $10 \leq \mathrm{h}<20 \mathrm{~km}$

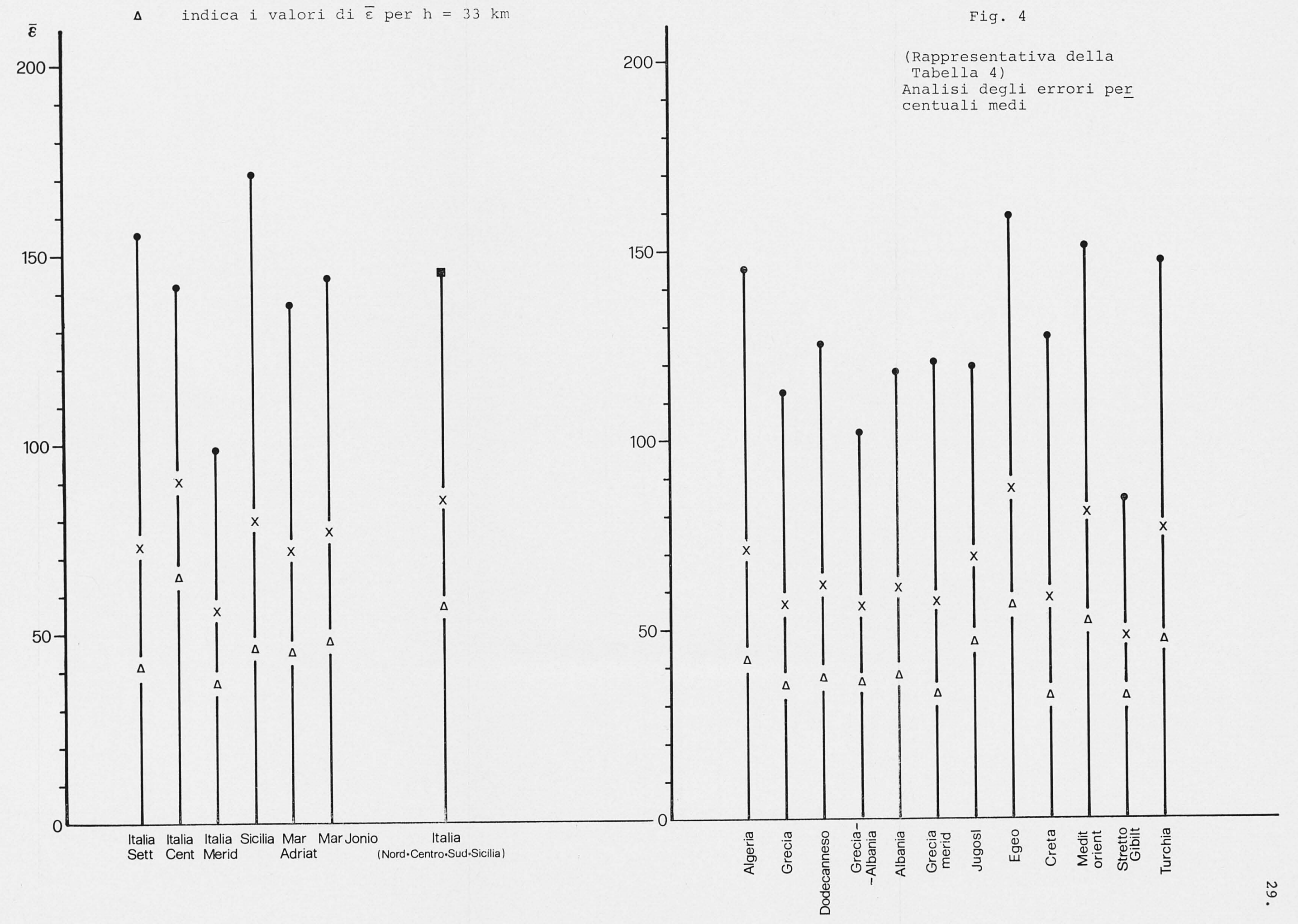

